\title{
MODIFICAÇÃO DE MEMBRANA DE POLIAMIDA VIA SOL-GEL E INCORPORAÇÃO DE COMPOSTO DE EURÓPIO (III) LUMINESCENTE
}

\author{
Érica A. de Souza a, Beatriz M. de Camposa , Lucas A. Rocha ${ }^{a}$, Emerson H. de Faria ${ }^{a}$, Katia J. Ciuffia, Eduardo J. Nassar ${ }^{a, *}$, \\ Jorge V. L. Silva ${ }^{\mathrm{b}}$, Marcelo F. Oliveira ${ }^{\mathrm{b}}$ e Izaque A. Maia ${ }^{\mathrm{b}}$ \\ aUniversidade de Franca, Av. Dr. Armando Salles Oliveira, 201 Pq. Universitário, 14046-000 Franca - SP, Brasil \\ ${ }^{b}$ Centro da Tecnologia da Informação Renato Archer (CTI) - Rod. Dom Pedro I, Km 143,6, 13069-901 Campinas - SP, Brasil
}

Recebido em 21/01/2016; aceito em 19/05/2016; publicado na web em 13/07/2016

\begin{abstract}
MODIFICATION OF POLYAMIDE MEMBRANE BY SOL-GEL AND INCORPORATION OF LUMINESCENT EUROPIUM (III) COMPLEX. Over the last decades, the combination of different technologies to search for systems with new properties and features has brought various segments of biological and earth sciences together. Additive manufacturing, known as rapid prototyping, combined with the sol-gel methodology enables the production of novel systems with applications in many scientific fields. In this work, flexible polyamide membranes were obtained by additive manufacturing, functionalized by the sol-gel methodology, and incorporated with the coordination compound between $\mathrm{Eu}(\mathrm{III})$ and 1,10-phenanthroline. The presence of vibrations at $1100 \mathrm{~cm}^{-1}$ in the FTIR spectrum of the material, which is a band typical of the Si-O-Si group in the alkoxide employed during the process, confirmed the polyamide membrane functionalization. The thermogravimetric curve showed that a residue remained after heating at $700{ }^{\circ} \mathrm{C}$, which was attributed to $\mathrm{SiO}_{2}$. The membrane was highly luminescent, which confirmed incorporation of the $\mathrm{Eu}^{3+}$ compound into the material and pointed to the possible application of this system as a topical medication for the treatment of skin diseases.
\end{abstract}

Keywords: sol-gel; photodynamic therapy; luminescence; europium III compounds.

\section{INTRODUÇÃO}

A terapia fotodinâmica (TFD) é uma técnica aplicada no tratamento de câncer de pele e que utiliza a luz (radiação eletromagnética) para tal. Resumidamente, a área tratada é exposta a radiação monocromática, região do visível (600 à 800 nm), após a administração de um fotossensibilizador que absorve a radiação e em seguida transfere a energia ao oxigênio molecular, gerando espécies reativas de oxigênio e, consequentemente, a oxidação dos lipídios, aminoácidos e proteínas, o que resulta na destruição do tumor. ${ }^{1,2}$ A técnica TFD utiliza emulsões, microemulsões, nanoemulsões, que são aplicadas diretamente sobre a pele e, após um determinado tempo, são absorvidas pelas células tumorais. A literatura tem relatado a utilização de vários sistemas que contém nanopartículas e fotossensibilizados..$^{3-5}$

Os elementos lantanídeos apresentam, como uma de suas principais carcterísticas, a propriedade fotoluminescentes, amplamente aplicadas na geração de radiação eletromagnética nas regiões do visível e infravermelho. As transições interconfiguracionais $f$ - $f$ são as responsáveis por essas propriedades ${ }^{6-8}$ Os íons lantanídeos podem sofrer excitação nas regiões do ultravioleta, visível e infravermelho, o que resulta em linhas de emissão monocromáticas na região do visível e do infravermelho. Por exemplo, o íon $\mathrm{Eu}^{3+}$ apresenta intensa emissão na região vermelha do espectro eletromagnético, aproximadamente em $620 \mathrm{~nm}$, quando excitado no ultravioleta (394 nm) e visível (460 $\mathrm{nm}) .{ }^{9-11}$ A combinação de elementos adequados também pode apresentar alta eficiência de emissão na região do visível quando se utiliza a radiação infravermelha, chamado de conversão ascendente, como por exemplo o par $\mathrm{Yb}^{3+}-\mathrm{Tm}^{3+} .{ }^{12}$ Portanto, os íons lantanídeos apresentam características para serem utilizados como emissores de radiação na região visível entre 600 e $800 \mathrm{~nm}$, atuando como fotossensibilizador e com possibilidade de aplicação na terapia fotodinâmica.

A Manufatura Aditiva (MA), também conhecida como Prototipagem Rápida (PR), é um conjunto de tecnologias utilizadas

*e-mail: eduardo.nassar@unifran.edu.br para a produção de peças complexas, que são construídas camadas-por-camadas. ${ }^{13}$ A Sinterização Seletiva a Laser (SLS) é uma das técnicas da MA que utiliza pó de poliamida como matéria prima para produzir peças através da sinterização do pó pelo laser. ${ }^{14}$

Pensando em unir as tecnologias, SLS e sol-gel, atrelando às propriedades espectroscópicas dos íons lantanídeos, esse trabalho teve como principal objetivo modificar as membranas flexíveis de poliamida obtidas por SLS e a elas incorporar compostos luminescentes para possíveis aplicações na terapia fotodinâmica. As membranas foram caracterizadas por difração de raios X, espectroscopia vibracional na região do infravermelho, análise térmica e fotoluminescência.

O diferencial dessa proposta é utilizar a membrana de poliamida modificada via metodologia sol-gel para suportar agentes senssibilizadores como os íons e os compostos de terras raras por meio de suas propriedades de emissão de luz após absorção de radiação na região do visível e infravermelho.

\section{PARTE EXPERIMENTAL}

\section{Preparação das Membranas}

\section{Lavagem das membranas}

A membrana de poliamida (PA), Figura 1, obtida por MA na divisão de Tecnologias Tridimensionais do Centro de Tecnologia da Informação Renato Archer, possui dimensões de 7,0 × 5,0 cm e espessura de $200 \mu \mathrm{m}$. A membrana de PA foi dividida em unidades com área de $1,0 \mathrm{~cm}^{2}$, com massa aproximada de $25,30 \mathrm{mg}$, que foram previamente lavadas em $30,0 \mathrm{~mL}$ de água destilada, sob agitação por $1 \mathrm{~h}$, para retirada do pó de poliamida, que não sofreu sinterização. Após a lavagem, as membranas foram secas à temperatura ambiente.

\section{Pré-Tratamento com ácido acético $1,0 \mathrm{~mol} \mathrm{~L}^{-1}$}

As membranas sofreram pré-tratamento, conforme descrito na literatura. ${ }^{15}$ Para tanto, foram mergulhadas em solução $1,0 \mathrm{~mol} \mathrm{~L}-1$ de ácido acético, sob agitação por $24 \mathrm{~h}$. As mesmas foram lavadas 


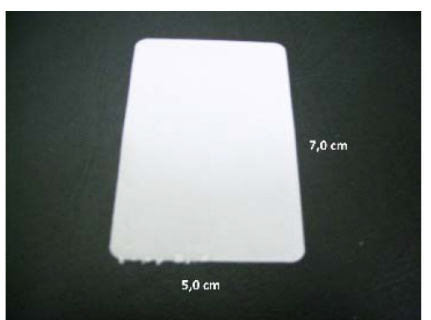

a

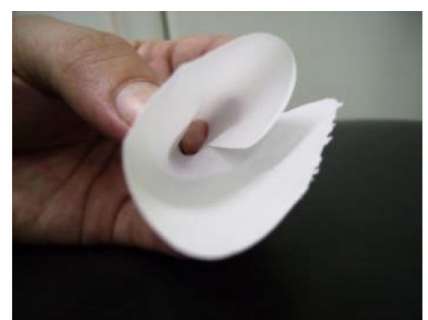

b

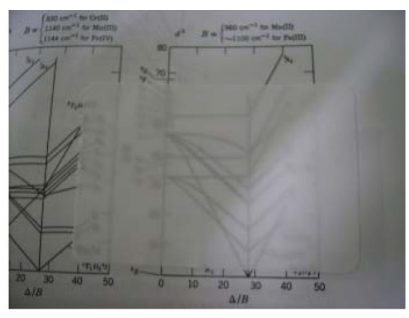

C

Figura 1. Foto da membrana de poliamida preparada por manufatura aditiva, a) dimensões da membrana, b) flexibilidade, c) transparência

com água destilada em banho de ultrassom e secas em temperatura ambiente por mais $24 \mathrm{~h}$. Esse tratamento foi realizado a fim de ativar a superfície da membrana pela protonação dos grupos NH da poliamida, o que facilita a interação da membrana com os alcóxidos.

\section{Modificação da membrana com os alcóxidos tetraetilortosilicato} (TEOS) e 3-cloropropiltrietoxisilano (CPTES)

As membranas de PA com área de $1,0 \mathrm{~cm}^{2}$ foram colocadas em um balão de fundo redondo de duas bocas contendo $15,0 \mathrm{~mL}$ de etanol (EtOH), 3,7 mL de TEOS e/ou 3,4 mL de CPTES e 5,0 $\mathrm{mL}$ de água destilada. A mistura foi mantida em temperatura de 80 ${ }^{\circ} \mathrm{C}$, sob refluxo e agitação constante por $24 \mathrm{~h}$. Após esse processo, as membranas foram lavadas com água destilada em um banho de ultrassom e secas a $60^{\circ} \mathrm{C}$ por $2 \mathrm{~h}$. As amostras foram designadas de PA TEOS E PA CPTES.

\section{Modificação da amostra PA TEOS com CPTES}

As membranas PA, após serem funcionalizadas com TEOS, sofreram funcionalização com CPTES, nas mesmas quantidades utilizadas isoladamente $(3,4 \mathrm{~mL}$ de CPTES), e assim obteve-se a amostra designada de PA TEOS CPTES.

Preparo do complexo [Eu(phen $\left.)_{2}\left(\mathrm{H}_{2} \mathrm{O}\right)_{2}\right] \mathrm{Cl}_{3}$

O complexo de európio III foi preparado como descrito na literatura, ${ }^{16} 200 \mathrm{mg}$ de 1,10-fenantrolina foram adicionados à $10,0 \mathrm{~mL}$ de EtOH seguida da adição de 2,0 mL da solução etanólica $0,1 \mathrm{~mol} \mathrm{~L}^{-1}$ de $\mathrm{EuCl}_{3}$. A mistura permaneceu sob agitação por $1 \mathrm{~h}$, adicionou-se $18,0 \mathrm{~mL}$ de acetona na mesma e o precipitado foi filtrado, lavado e seco com temperatura de $50{ }^{\circ} \mathrm{C}$ e pressão reduzida por $4 \mathrm{~h}$. A análise termogravimétrica confirmou a formação do composto com a fórmula molecular $\left[\mathrm{Eu}(\text { phen })_{2}\left(\mathrm{H}_{2} \mathrm{O}\right)_{2}\right] \mathrm{Cl}_{3}$.

\section{Incorporação do complexo nas membranas PA funcionalizada}

As membranas modificadas foram colocadas em contato com a solução etanólica do complexo $\left[\mathrm{Eu}(\text { phen })_{2}\left(\mathrm{H}_{2} \mathrm{O}\right)_{2}\right] \mathrm{Cl}_{3}$. A mistura foi mantida sob agitação constante por $1 \mathrm{~h}$ e após esse processo, as membranas foram lavadas com água destilada em um banho de ultrassom e secas a $60{ }^{\circ} \mathrm{C}$ por $2 \mathrm{~h}$. A amostra foi designada de PA CPTES Compl e PA TEOS CPTES Compl.

\section{Caracterizações}

Difratometria de raios $X(D R X)$

Os difratoframas de raios $X$ foram obtidos em temperatura ambiente em um difratômetro Rigaku Geigerflex D/Max-c, com monocromador de radiação $\mathrm{CuK} \alpha(\lambda=1,5405 \AA)$, num intervalo de $2 \theta$ entre de 5 a $70^{\circ}$ com passo de $0,02^{\circ} / 10 \mathrm{~s}$.

Análises térmicas (TG e DSC)

As análises térmicas TG e DSC foram obtidas em um aparelho TA Instruments-SDT Q600-Simultaneous DTA-TGA, por meio da utilização de um gradiente de temperatura, que compreende desde a temperatura ambiente $\left(25^{\circ} \mathrm{C}\right)$ até $700{ }^{\circ} \mathrm{C}$, a uma velocidade de 20 ${ }^{\circ} \mathrm{C} / \mathrm{min}$, com o fluxo de nitrogênio de $100 \mathrm{~mL} / \mathrm{mim}$.

Espectroscopia de absorção na região do infravermelho (FTIR)

Para as análises de FTIR, foi utilizado um espectrofotômetro PerkinElmer Frontier com faixa de operação entre 400-4000 cm-1. O espectro de absorção foi realizado por ATR (Espectroscopia de absorção no FTIR por refletância total atenuada), com varredura de 16 scans.

\section{Fotoluminescência $(F L)$}

Os espectros de excitação e emissão foram realizados em um espectrofluorímetro FluoroLog - Horiba Jobin Yvon. As medidas foram coletadas a $90^{\circ}$ em relação ao feixe incidente, utilizando filtro de $500 \mathrm{~nm}$. As fendas de excitação $\left(f_{\text {exc }}\right)$ e de emissão $\left(f_{\text {em }}\right)$ foram 2,0 e $1,0 \mathrm{~nm}$, respectivamente.

\section{RESULTADOS E DISCUSSÃO}

\section{Difração de raios X (DRX)}

A poliamida, no estado sólido, pode apresentar duas formas semi-cristalinas, forma $\alpha$ e $\gamma \cdot{ }^{17} \mathrm{~A}$ forma $\alpha$ é caracterizada pela célula unitária triclínica com configuração planar em zig-zag, e apresentou dois picos no difratograma de raios $\mathrm{X}, 2 \theta=20,5$ e $24^{\circ}$ e a forma $\gamma$ pseudohexagonal caracterizada por pico em $2 \theta=21,5^{\circ} \cdot{ }^{18,19}$ A Figura 2 apresenta os DRX das membranas de poliamida após tratamento e modificação com os alcóxidos.

As ligações de hidrogênio entre as cadeias de poliamida são responsáveis pelas formas amorfas e semicristalinas do polímero.

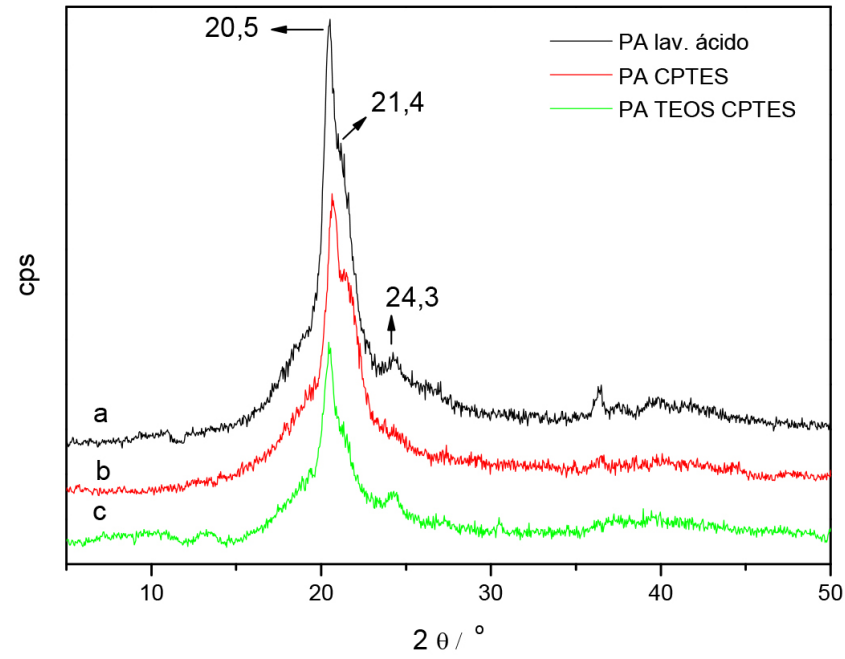

Figura 2. DRX para as membranas PA a) lavada com ácido acético, b) modificada com CPTES e c) modificada com TEOS e CPTES 
As semicristalinas apresentam picos no DRX em $2 \theta=20,5(\mathrm{~d}=$ $0,43 \mathrm{~nm})$ e $24,3^{\circ}(\mathrm{d}=0,37 \mathrm{~nm})$ para a forma $\alpha$ e $21,5^{\circ}(\mathrm{d}=0,41$ nm) para a forma $\gamma^{20-22}$ os mesmos observados nos difratogramas da Figura $2, \theta=20,2^{\circ}(\mathrm{d}=0,44 \mathrm{~nm}), \theta=23,4^{\circ}(0,37 \mathrm{~nm})$ e $\theta=$ $21,3^{\circ}(0,42 \mathrm{~nm})$. A razão da intensidade dos picos correspondente às formas $\alpha$ e $\gamma$ pode indicar o grau de conversão de uma forma na outra. A Figura 2 mostrou que as proporções entre os picos permaneceram constante para todas as membranas, indicando que o tratamento e modificação não afetaram as ligações de hidrogênio. A transição no estado sólido ocorre principalmente através da ação da temperatura e é caracterizada pela mudança da forma cristalina triclínica $(\alpha)$ para a pseudohexagonal $(\gamma)$, caracterizada pela convergência de dois fortes sinais difração de raios $\mathrm{X}$, para um valor intermediário. A mudança pode ser atribuída a um parcial rearranjo das ligações de hidrogênio entre as cadeias de hidrocarboneto. ${ }^{21,23}$ Esse fato pode ser confirmado pela espectroscopia vibracional na região do infravermelho, no qual as bandas em $934 \mathrm{~cm}^{-1}$ atribuídas ao estiramento C-CO e em $1202 \mathrm{~cm}^{-1}$, a banda característica da interação de amida III com o esqueleto hidrocarboneto, indicam a existência das fases cristalinas..$^{23,24}$

Os DRXs das membranas modificadas após a adição do complexo de európio com 1,10-fenantrolina $\left[\mathrm{Eu}(\text { phen })_{2} \cdot\left(\mathrm{H}_{2} \mathrm{O}\right)_{2}\right] \mathrm{Cl}_{3}$ apresentaram as mesmas razões entre os picos das formas $\alpha$ e $\gamma$, indicando que o complexo também não influenciou na ligações de hidrogênio das cadeias.

\section{Espectroscopia vibracional (FTIR)}

A dissociação do ácido acético em água permite a liberação de prótons, os quais podem protonar os grupamentos - $\mathrm{NH}$ da poliamida, produzindo uma carga parcial positiva. ${ }^{25,26}$ Os espectros vibracionais mostraram que a região - $\mathrm{NH}$ da membrana de poliamida sem prévio tratamento apresenta banda em $1537 \mathrm{~cm}^{-1}$ e após tratado com ácido acético essa banda desloca para $1556 \mathrm{~cm}^{-1}$ e essa diferença pode ser atribuída a protonação dos grupos - $\mathrm{NH}$. Na membrana tratada com ácido e lavada com água, observou-se banda com o mesmo número de onda $\left(1556 \mathrm{~cm}^{-1}\right)$, indicando que a desprotonação não ocorreu. $\mathrm{O}$ espectro vibracional para as amostras modificada apresentou um deslocamento da banda, nesta região para $1566 \mathrm{~cm}^{-1}$, indicativo que interações podem ocorrer por meio dos grupos amidas do polímero com os agentes modificadores TEOS e CPTES.

Os espectros vibracionais na região do infravermelho apresentaram os principais modos vibracionais da poliamida, antes e após o tratamento com ácido acético, o que indica que o tratamento não promoveu alterações nas formas semicristalinas da poliamida que compõem a membrana, o que confirma o observado pela difração de raios X. Os espectros apresentam as vibrações características da deformação -NH e estiramento -CN de amida em 1556, 1639 e $3295 \mathrm{~cm}^{-1}$. As bandas na região de 2846 e $2924 \mathrm{~cm}^{-1}$ se devem às vibrações - $\mathrm{CH}_{2},-\mathrm{CH}_{3}$ e NH e as bandas em 727, 1463,1559 e $1639 \mathrm{~cm}^{-1}$ podem ser atribuídas aos grupos $\mathrm{N}-\mathrm{H}, \mathrm{C}-\mathrm{CO}-\mathrm{NH}$ e $\mathrm{C}=\mathrm{O}$ da poliamida. $^{27,28}$

A Figura 3 apresenta os espectros FTIR das membranas de poliamida antes e após sofrerem modificações com os alcóxidos CPTES e TEOS CPTES.

A Figura 3 apresenta os espectros vibracionais das membranas e as bandas características da poliamida são observadas antes e após a modificação com os alcóxidos. Uma diminuição na intensidade das bandas para a amostra modificada com TEOS CPTES pode ser decorrente de um maior recobrimento da membrana pelos alcóxidos. Novas bandas surgiram no espectro das amostras modificadas, bandas atribuídas aos alcóxidos, comprovando a modificação da membrana. Vibrações da ligação de $\mathrm{C}$-Cl podem ser observadas na região entre

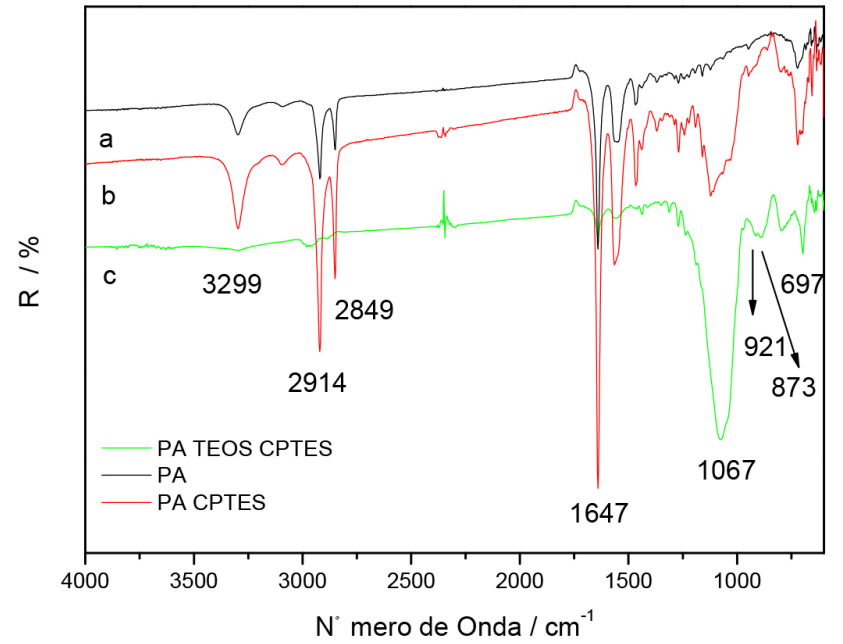

Figura 3. FT-IR da membrana de PA (a), modificada com CPTES (b) e TEOS CPTES (c)

698 e $695 \mathrm{~cm}^{-1} \cdot{ }^{29,30}$ As bandas em 873 e $1279 \mathrm{~cm}^{-1}$ são atribuídas às deformações C-Si presente no alcóxido CPTES. A região de 1067 $\mathrm{cm}^{-1}$ pode ser associada ao estiramento dos grupos siloxanos presentes na rede de silício (Si-O-Si), ${ }^{31,32}$ e em $923 \mathrm{~cm}^{-1}$ que está associada ao estiramento simétrico dos grupos siloxanos ( $\mathrm{Si}-\mathrm{O}-\mathrm{Si})$ da rede inorgânica. ${ }^{33,34}$

Os espectros das amostras após a incorporação do complexo $\left[\mathrm{Eu}(\text { phen })_{2} \cdot\left(\mathrm{H}_{2} \mathrm{O}\right)_{2}\right] \mathrm{Cl}_{3}$, apresentaram bandas de absorção em $1426 \mathrm{e}$ $1425 \mathrm{~cm}^{-1}$ atribuídos aos estiramento $\mathrm{C}=\mathrm{C}$ e $\mathrm{C}=\mathrm{N}$ do anel, presentes na 1,10-fenantrolina. ${ }^{35,36}$ Essas bandas são observadas no espectro do complexo e nas amostras as quais foram incorporados o composto de coordenação. As bandas em 1583 e $1520 \mathrm{~cm}^{-1}$ podem ser atribuídas à vibração do esqueleto aromático das ligações $\mathrm{C}=\mathrm{C}$ e duas bandas em 851 e $733 \mathrm{~cm}^{-1}$ indicam deformações fora do plano da ligação C-H do anel aromático da molécula de 1,10-fenantrolina. ${ }^{36}$

A banda em $1556 \mathrm{~cm}^{-1}$, atribuída à forma protonada do grupo -NH da poliamida $\left(-\mathrm{NH}_{2}^{+}\right)$desloca-se para $1566 \mathrm{~cm}^{-1}$ após a modificação, o que pode ser um indício de que a interação entre os grupos silanóis $(\mathrm{Si}-\mathrm{OH})$, formados a partir dos alcóxido, e $-\mathrm{NH}_{2}{ }^{+}$podem estar ocorrendo.

O íon európio III e os grupos -NH (poliamida) e $\mathrm{ROH}$ (dos alcóxidos) são classificados como ácido e bases duras, respectivamente, segundo a definição de Pearson. ${ }^{37}$ Segundo essa teoria, o ácido duro reage preferencialmente com base dura, assim, a interação entre o complexo pode ocorrer diretamente com a membrana de poliamida ou com os grupos silanóis da sílica. A Figura 4 apresenta a ampliação no espectro vibracional das membranas modificadas com CPTES e TEOS CPTES, antes e após a incorporação do complexo da região do anel aromático do ligante phen e da região de protonação do grupo -NH da poliamida.

\section{Análises térmicas (TG/DSC)}

As análises térmicas foram realizadas a fim de avaliar o comportamento térmico envolvido nas modificações sofridas pela membrana de poliamida, bem como comprovar a adição dos alcóxidos na mesma. A Figura 5 apresenta as curvas termogravimétricas das membranas antes e após tratamento com ácido acético e modificação.

As análises térmicas mostraram apenas uma perda de massa entre 300 e $550{ }^{\circ} \mathrm{C}$ para as membranas e que está associada à degradação completa do polímero (poliamida). As curvas termogravimétricas para as membranas modificadas com CPTES e TEOS CPTES apresentaram resíduos, atribuídos à formação da rede de sílica proveniente das 


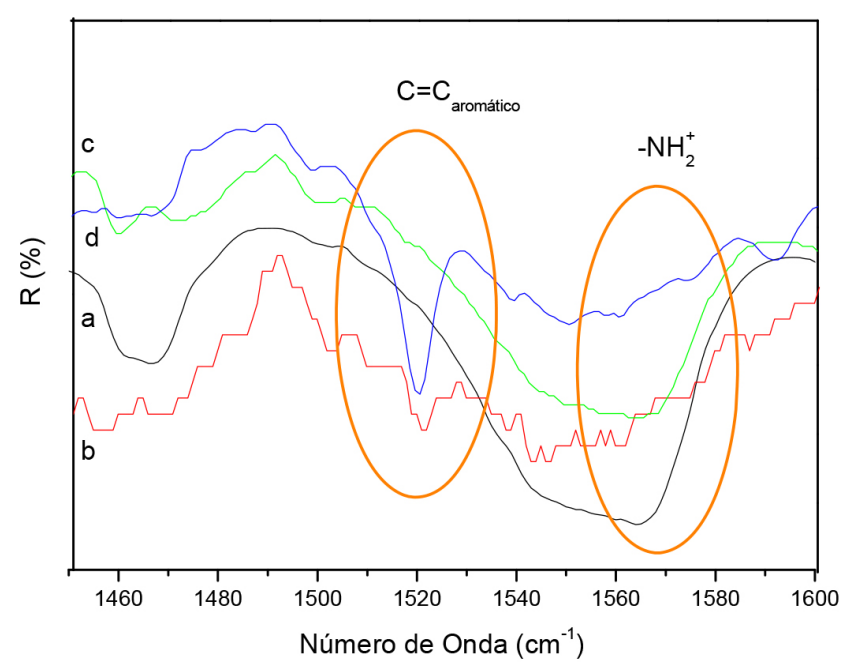

Figura 4. FT-IR da membrana de PA modificada com CPTES (a), CPTES Compl. (b), TEOS CPTES (c) e TEOS CPTES Compl. (d)

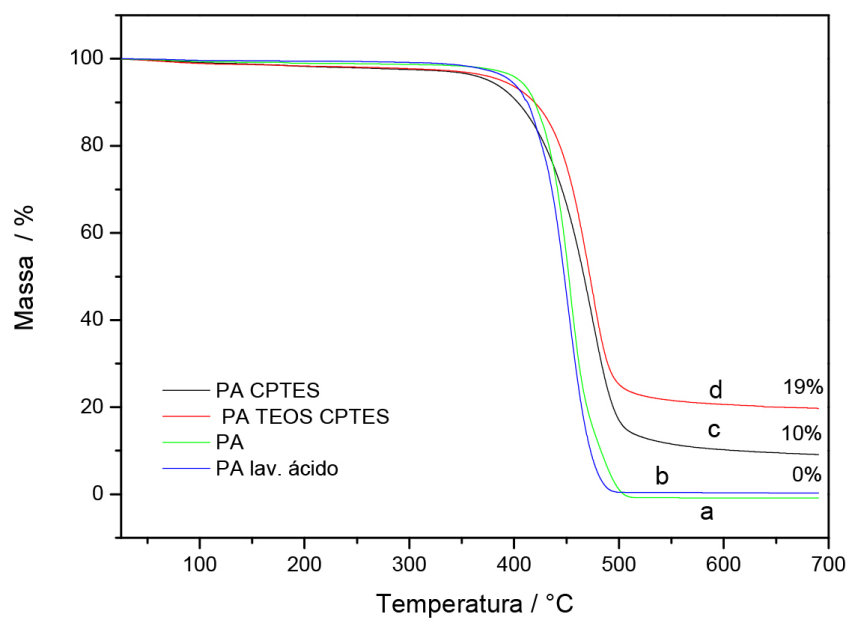

Figura 5. Curvas termogravimétricas $(T G)$ da membrana pura (a), tratada com ácido acético (b), modificada com os alcóxidos, PA CPTES (c) e PA TEOS CPTES $(d)$

reações de hidrólise e condensação dos alcóxidos. Para a membrana modificada com CPTES, o resíduo após $700{ }^{\circ} \mathrm{C}$ foi de $10 \%$ e para a membrana modificada com TEOS-CPTES, esse resíduo foi de $19 \%$. O resíduo após tratamento térmico é essencialmente a formação do óxido $\left(\mathrm{SiO}_{2}\right)$, desta forma, a curva termogravimétrica indica a presença de resíduos inorgânicos na membrana, o que indica a presença dos agentes modificadores (alcóxidos), fato constatado anteriormente através dos espectros de FTIR. As curvas DTG para as membranas apresentaram aumento na estabilidade térmica da poliamida após sofrerem o revestimento. A Tabela 1 apresenta o intervalo de temperatura de decomposição da membrana de poliamida, resíduo e a temperatura da máxima taxa de decomposição.

$\mathrm{O}$ aumento na estabilidade térmica da poliamida após revestimento via sol-gel já havia sido observado em trabalhos anteriores. ${ }^{15,34,38}$

Segundo Ballistreri et al., ${ }^{39}$ a degradação das poliamidas, em geral, ocorre em temperaturas superiores a $400{ }^{\circ} \mathrm{C}$ e, neste trabalho, a degradação ocorreu em temperaturas acima de $450^{\circ} \mathrm{C}$ (Tabela 1), esse aumento pode estar relacionado às formas $\alpha$ e $\gamma$ e o número de ligações de hidrogênio entre as cadeias. ${ }^{40,41}$ As membranas modificadas com CPTES e TEOS CPTES apresentaram temperaturas superiores às membranas não modificadas, indicativo de mudanças na propriedade térmica da PA por meio da incorporação dos alcóxidos.
Tabela 1. Intervalo de temperatura de decomposição da matéria orgânica, resíduo inorgânico e temperatura da máxima taxa de decomposição para as membranas obtido através das curvas DTG

\begin{tabular}{lccc}
\hline Amostra & $\begin{array}{c}\text { Intervalo de tem- } \\
\text { peratura }\left({ }^{\circ} \mathrm{C}\right)\end{array}$ & $\begin{array}{c}\text { Resíduo de } \\
\text { massa }(\%)\end{array}$ & $\begin{array}{c}\text { Temperatura da } \\
\text { máxima taxa de } \\
\text { decomposição } \\
\left({ }^{\circ} \mathrm{C}\right)\end{array}$ \\
\hline P.A & $370-502$ & 0 & 450 \\
PA lav. ácido & $368-553$ & 0 & 453 \\
PA CPTES & $351-536$ & 10 & 474 \\
PA TEOS CPTES & $333-536$ & 19 & 475 \\
\hline
\end{tabular}

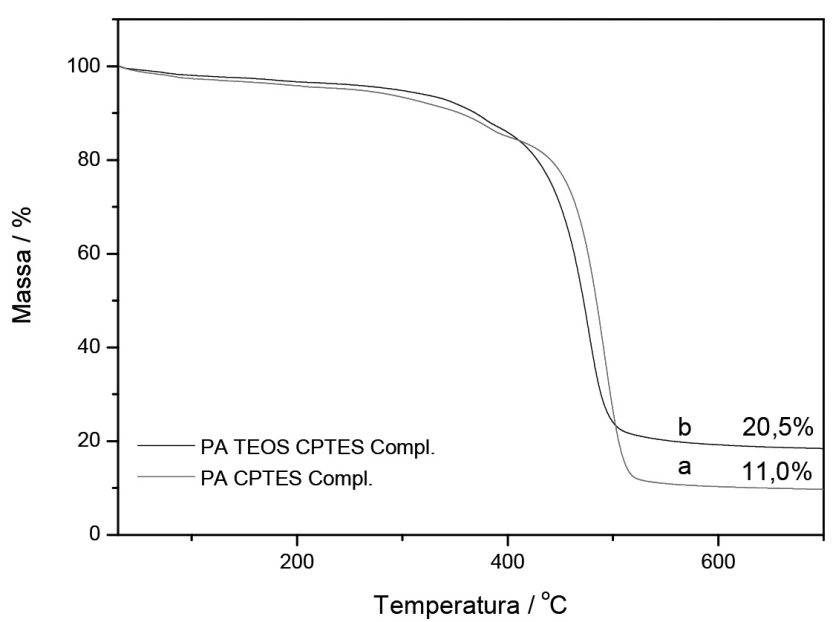

Figura 6. Curvas termogravimétricas (TG) das membranas modificadas após incorporação do complexo, (a) PA CPTES Compl. e (b) PA TEOS CPTES Compl.

A Figura 6 apresenta as curvas termogravimétricas para as membranas após a incorporação do complexo.

As curvas termogravimétricas apresentaram perfis diferentes com relação às curvas das membranas modificadas (Figura 5). As TGs apresentaram início de perda de massa abaixo de $300{ }^{\circ} \mathrm{C}$, também verificados nas curvas DTG. Observou-se um aumento no resíduo de cerca de $1 \%$ para as duas amostras e tal aumento é atribuído, provavelmente, à formação de óxido de európio III, produto da decomposição do complexo $\left[\mathrm{Eu}(\text { phen })_{2}\left(\mathrm{H}_{2} \mathrm{O}\right)_{2}\right] \mathrm{Cl}_{3}$. A temperatura da máxima taxa de decomposição pode ser obtida através da curva DTG, que apresentou um aumento de $474{ }^{\circ} \mathrm{C}$ da membrana modificada com CPTES para $483^{\circ} \mathrm{C}$ após a adição do complexo, e de 475 ${ }^{\circ} \mathrm{C}$ para $477{ }^{\circ} \mathrm{C}$ para a membrana modificada com TEOS CPTES e após a adição do complexo, respectivamente. Os dados da análise térmica confirmam o observado pela espectroscopia vibracional e fotoluminescência.

A Figura 7 apresenta as curvas de Calorimetria Exploratória Diferencial (DSC) para as membranas de poliamida e após a incorporação do complexo.

As curvas DSC apresentaram transição vítrea $(\mathrm{Tg})$ próximas ao descrito na literatura,,$^{15}$ entre 70 e $90{ }^{\circ} \mathrm{C}$. A região com temperatura ao redor de $190{ }^{\circ} \mathrm{C}$ corresponde à temperatura de fusão (Tf) da PA antes e após a modificação e incorporação do complexo. A temperatura de decomposição (TdPA) da PA ocorre ao redor de $470{ }^{\circ} \mathrm{C}$ e apresentou deslocamento, o que indica influência dos alcóxidos na decomposição da PA. As membranas que tiveram o complexo de európio III incorporado apresentaram pico ao redor de $400{ }^{\circ} \mathrm{C}$ atribuído à decomposição do complexo (TdCompl.), ${ }^{42}$ esses resultados corroboram os resultados da FTIR, TG e DTG. 


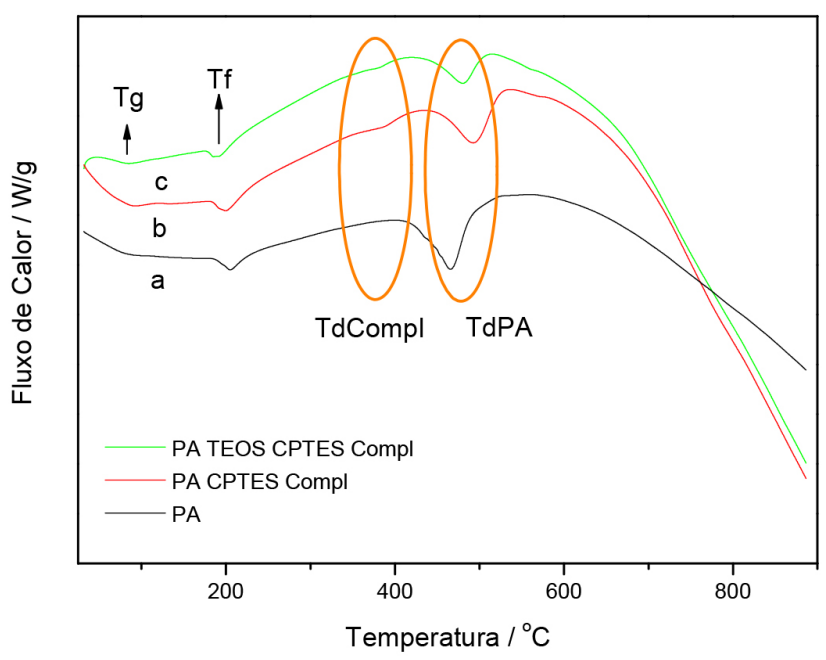

Figura 7. Curvas DSC para a membrana de PA (a), modificada e após incorporação do complexo, (b) PA CPTES Compl. e (c) PA TEOS CPTES Compl.

\section{Fotoluminescência (FL)}

Os espectros de excitação apresentam uma larga banda entre 280 - $400 \mathrm{~nm}$, atribuída à banda de transferência de carga ligante-metal (BTC), ${ }^{43-46}$ com máximos em $356 \mathrm{~nm}$ para o complexo $\left[\mathrm{Eu}(\text { phen })_{2}\left(\mathrm{H}_{2} \mathrm{O}\right)_{2}\right] \mathrm{Cl}_{3}, 335$ e $347 \mathrm{~nm}$ para as amostras PA CPTES Compl e PA TEOS CPTES Compl, respectivamente.

As linhas $f$ - $f$ do íon $\mathrm{Eu}^{3+}$ referente às transições ${ }^{7} \mathrm{~F}_{0} \rightarrow{ }^{5} \mathrm{~L}_{6}(394$ $\mathrm{nm})$ e ${ }^{7} \mathrm{~F}_{0} \rightarrow{ }^{5} \mathrm{D}_{2}(465 \mathrm{~nm})$ foram observadas para o complexo, para as membranas contendo o complexo, essas transições aparecem com menor intensidade.

A Figura 8 mostra os espectros de emissão do complexo $\left[\mathrm{Eu}(\text { phen })_{2}\left(\mathrm{H}_{2} \mathrm{O}\right)_{2}\right] \mathrm{Cl}_{3}$ e dele incorporado às membranas, PA CPTES Compl e PA TEOS CPTES Compl.

Os espectros de emissão apresentam as bandas características de

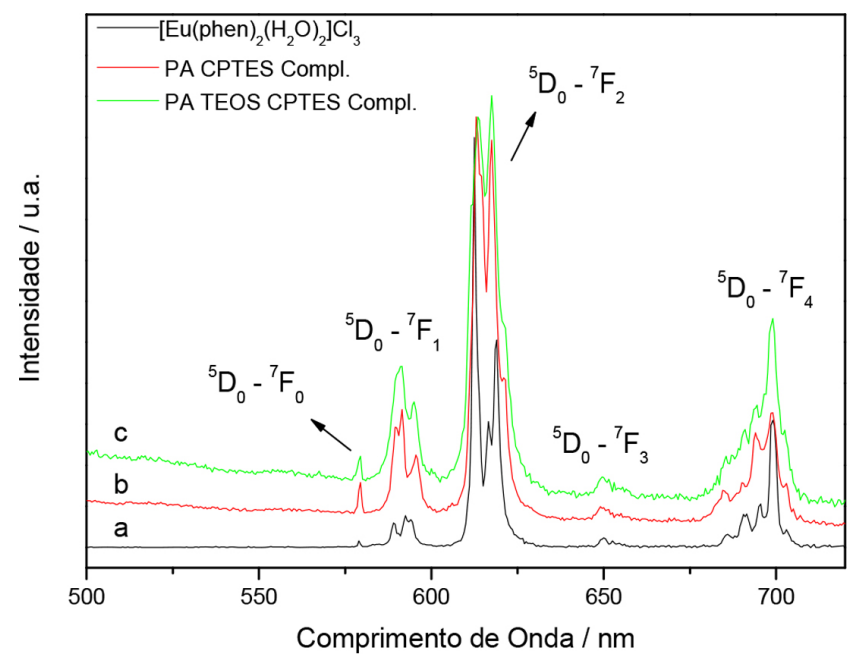

Figura 8. Espectros de emissão, a) $\left.\left[\mathrm{Eu}(\text { phen })_{2}\left(\mathrm{H}_{2} \mathrm{O}\right)_{2}\right] \mathrm{Cl}_{3}\left(\lambda_{\text {exc }}=356 \mathrm{~nm}\right), b\right)$ PA CPTES Compl $\left(\lambda_{\text {exc }}=335 \mathrm{~nm}\right)$ e c) PA TEOS CPTES Compl $\left(\lambda_{\text {exc }}=347 \mathrm{~nm}\right)$ emissão do íon Eu3+, correspondente às transições do estado excitado ${ }^{5} \mathrm{D}_{0}$ para o fundamental ${ }^{7} \mathrm{~F}_{\mathrm{J}}(\mathrm{J}=0,1,2,3$ e 4$)$, quando excitado na BTC e no nível ${ }^{5} \mathrm{~L}_{6}$ do íon. A Figura 8 apresenta os espectros de emissão quando excitado na BTC. A banda relativa à transição ${ }^{5} \mathrm{D}_{0}$ ${ }^{7} \mathrm{~F}_{0}$ está presente nos espectros, indicando que o íon $\mathrm{Eu}^{3+}$ ocupa sítio de simetria sem centro de inversão. ${ }^{47} \mathrm{~A}$ semelhança entre os espectros de emissão do complexo nas membranas em relação ao complexo isolado é mais um indicativo da incorporação do complexo nas membranas de poliamida modificadas, fato confirmado pelas outras técnicas de caracterização. Em prévio trabalho, a incorporação de complexo de európio III em peças de Acrilonitria-Butadieno-Estireno (ABS) obtidas por manufatura aditiva também foi confirmada através da espectroscopia luminescente. ${ }^{48}$

O espectro de emissão é um indicativo de que não houve uma grande mudança no ambiente de simetria do íon. Comparando o número de bandas teóricas ${ }^{49} \mathrm{com}$ as experimentais, tem-se a indicação que o íon $\mathrm{Eu}^{3+}$ ocupa um sítio com o grupo pontual $\mathrm{C}_{2 \mathrm{v}}$. A mudança na energia da transição ${ }^{5} \mathrm{D}_{0} \rightarrow{ }^{7} \mathrm{~F}_{0}$ pode indicar uma mudança no campo cristalino ao redor do íon $\mathrm{Eu}^{3+}$, indicando uma mudança no grau de covalência da ligação Eu-O. ${ }^{50}$ Neste trabalho, observou-se uma mudança para baixa energia da transição ${ }^{5} \mathrm{D}_{0} \rightarrow{ }^{7} \mathrm{~F}_{0}$ quando comparada com o complexo isolado, sugerindo um aumento no grau de covalência da ligação após a incorporação do complexo na membrana, indicativo da interação complexo-membrana, esse comportamento também foi observado quando Rocha et al. ${ }^{51}$ incorporou compostos de $\mathrm{Eu}^{3+}$ em sílica mesoporosa, o mesmo foi observado por Azevedo et al. ${ }^{46}$

A Tabela 2 apresenta a razão entre as intensidades relativas das transições (AR) $\mathrm{AR}\left({ }^{5} \mathrm{D}_{0} \rightarrow{ }^{7} \mathrm{~F}_{2}\right) / \mathrm{AR}\left({ }^{5} \mathrm{D}_{0} \rightarrow{ }^{7} \mathrm{~F}_{1}\right)$, os parâmetros de intensidade Judd-Ofelt $\left(\Omega_{2}\right.$ and 4$)$, os decaimentos radiativos e não-radiativos $\left(\mathrm{A}_{\mathrm{rad}}, \mathrm{A}_{\mathrm{nrad}}\right)$, o tempo de vida experimental $(\tau)$ e a eficiência quântica $(\eta)$ de emissão do íon $\mathrm{Eu}^{3+}$, calculados a partir dos espectros de emissão com ajuda do programa LUMPAC (Lanthanide Luminescence Software). ${ }^{52}$

A literatura considera que o parâmetro $\Omega_{2}$ pode indicar a covalência da ligação entre os íons lantanídeos e o retículo, maiores valores de $\Omega_{2}$ mais covalente é a ligação e menor a simetria ao redor do íon $\mathrm{Eu}^{3+} .53,54$ Na Tabela 2, pode-se observar que os valores de $\Omega_{2}$ para o complexo incorporado à membrana diminui em relação ao complexo isolado, e isso é um indicativo da diminuição da covalência da ligação, indicando que o complexo está interagindo com os grupos existentes na superfície da membrana. A diminuição dos valores de $\Omega_{2}$ indica uma diminuição da simetria ao redor do íon, comprovada pela diminuição da razão entre as áreas das bandas relativas as transições 0 - 2/0 - 1 .

O parâmetro $\Omega_{4}$ pode significar a rigidez estrutural e ser influenciado pelas vibrações do sistema (íon e ligante). ${ }^{55}$ Os baixos valores dos parâmetros $\Omega_{2}$ e $\Omega_{4}$ sugere uma fraca polarizabilidade e um ambiente químico rígido ao redor do íon $\mathrm{Eu}^{3+}$, mais um indicativo da interação entre o complexo com a membrana modificada. ${ }^{56}$

Os parâmetros $A_{\text {rad }}, A_{\text {nrad }}$ e $\tau$ podem fornecer informações sobre a população do estado excitado e dos processos de decaimento radiativo e não-radiativo do íon. ${ }^{57} \mathrm{~A}$ Tabela 2 apresenta alterações nos valores de $\mathrm{A}_{\text {rad }}$, porém os valores de $\mathrm{A}_{\text {nrad }}$ apresentam maiores diferenças, a diminuição do $\mathrm{A}_{\text {nrad }}$ promove um aumento no tempo de vida da emissão e eficiência quântica, esses valores indicam que os complexos estão se ligando mais fortemente à membrana modificada.

Tabela 2. Intensidade Relativa $\left(\operatorname{AR}\left({ }^{5} \mathrm{D}_{0} \rightarrow{ }^{7} \mathrm{~F}_{2}\right) / \mathrm{AR}\left({ }^{5} \mathrm{D}_{0} \rightarrow{ }^{7} \mathrm{~F}_{1}\right)\right)$, parâmetros de intensidade Judd-Ofelt $\left(\Omega_{2 \mathrm{e} 4}\right)$, decaimento radiativo e não-radiativo $\left(\mathrm{A}_{\text {rad }}\right.$ e $\left.\mathrm{A}_{\text {nrad }}\right)$, tempo de vida experimental $(\tau)$ e eficiência quântica $(\eta)$

\begin{tabular}{|c|c|c|c|c|c|c|c|}
\hline Amostra & $0-2 / 0-1$ & $\Omega_{2} \times 10^{-20}\left(\mathrm{~cm}^{2}\right)$ & $\Omega_{4} \times 10^{-20}\left(\mathrm{~cm}^{2}\right)$ & $\mathrm{A}_{\mathrm{rad}}\left(\mathrm{s}^{-1}\right)$ & $A_{\text {nrad }}\left(s^{-1}\right)$ & $\tau(\mathrm{ms})$ & $\eta(\%)$ \\
\hline$\left[\mathrm{Eu}(\text { phen })_{2}\left(\mathrm{H}_{2} \mathrm{O}\right)_{2}\right] \mathrm{Cl}_{3}\left(\lambda_{\text {exc }}=356 \mathrm{~nm}\right)$ & 7,57 & 13,37 & 9,26 & 586,34 & 1739,24 & 0,43 & 25,21 \\
\hline PA CPTES Compl $\left(\lambda_{\mathrm{exc}}=335 \mathrm{~nm}\right)$ & 5,41 & 9,52 & 7,45 & 482,75 & 1055,71 & 0,65 & 31,38 \\
\hline PA TEOS CPTES Compl $\left(\lambda_{\mathrm{exc}}=347 \mathrm{~nm}\right)$ & 5,51 & 9,70 & 8,70 & 508,44 & 900,01 & 0,71 & 36,10 \\
\hline
\end{tabular}


O tempo de vida experimental pode fornecer informações com relação ao número de moléculas de água coordenada ao íon $\mathrm{Eu}^{3+}$, calculadas segundo a equação descrita por Binnemans. ${ }^{49} \mathrm{O}$ tempo de vida da emissão para o complexo $\left[\mathrm{Eu}(\text { phen })_{2}\left(\mathrm{H}_{2} \mathrm{O}\right)_{2}\right] \mathrm{Cl}_{3}$ é 0,43 ms, correspondendo a 2 moléculas de água, confirmando a fórmula proposta para o complexo através da curva TG. Após a incorporação do complexo nas membranas modificadas ocorreu um aumento para os tempos de vida, 0,65 e 0,71 para as amostras PA CPTES Compl e PA TEOS CPTES Compl, respectivamente, indicando a presença de apenas 1 molécula de água nos complexos. Esse fato pode explicar a diminuição do $\mathrm{A}_{\text {nrad }}$ e aumento da eficiência quântica.

\section{CONCLUSÃO}

A modificação da membrana de poliamida com alcóxidos de silício e posterior incorporação de compostos opticamente ativos, como os íons lantanídeos e seus complexos, abrem caminhos para aplicações envolvendo a emissão de radiação eletromagnética. A metodologia utilizada na modificação é versátil e pode ser utilizada para outros alcóxidos contendo diferentes grupos funcionais.

As técnicas envolvidas nas caracterizações indicaram a presença do alcóxido na membrana de poliamida, bem como a presença do complexo de európio III. A eficiência de emissão é um parâmetro importante para aplicação na terapia fotodinâmica, podendo reduzir o tempo de exposição do fotossensibilizador à fonte de excitação, consequentemente reduzindo a exposição do paciente à radiação. Sistemas contendo íons lantanídeos com excitação na região do visível e infravermelho e emissão na região utilizada na TFD são promissores para essa aplicação.

\section{AGRADECIMENTOS}

Os autores agradecem as agências de fomento CNPq, CAPES e a FAPESP pela bolsa de mestrado da E.A.S. 2013/20443-4.

\section{REFERENCIAS}

1. Lin, A.; Hahn, S. M.; Clin. Cancer. Res. 2009, 15, 4252.

2. Rossetti, F. C.; Lopes, L. B.; Carollo, A. R. H.; Thomazini, J. A.; Tedesco, A. C.; Bentley M. V. L. B.; J. Controlled Release 2011, 155, 400.

3. de Paula, L. B.; Primo, F. L.; Pinto, M. R.; Morais, P. C.; Tedesco, A. C.; J. Magn. Magn. Mater. 2015, 380, 372.

4. Vijayaraghavan, P.; Vankayala, R.; Chiang, C.-S.; Sung, H.-W.; Hwang, K. C.; Biomaterials 2015, 62, 13.

5. Bolfarini, G. C.; Siqueira-Moura, M. P.; Demets, G. J. F.; Tedesco, A. C.; Dyes Pigm. 2014, 100, 162.

6. Tong, B.-H.; Wang, S.-J.; Jiao, J.; Ling, F.-R.; Meng, Y.-Z.; Wang, B.; J. Photochem. Photobiol. A 2007, 191, 74

7. Blasse, G.; Grabmaier, B. C.; Luminescent Materials, Springer: Heidelberg, 1994.

8. Monteiro, M. A. F.; Brito, H. F.; Felinto, M. C. F. C.; Brito, G. E. S.; Teotonio, E. E. S.; Vichi, F. M.; Stefani, R., Microporous Mesoporous Mater. 2007, 108, 237.

9. Matias, C. R.; Nassar, E. J.; Verelst, M.; Rocha, L. A.; J. Braz. Chem. Soc. 2015, 26, 2558.

10. Freiria, G. S.; Nassar, E. J.; Dexpert-Ghys, J.; Verelst, M.; Rocha, L. A.; J. Lumin. 2016, 169, 844.

11. Nassar, E. J.; Ávila, L. R.; Pereira, P. F. S.; Mello, C,; de Lima, O. J.; Ciuffi, K. J.; Carlos, L. D.; J. Lumin. 2005, 111, 159.

12. Rodríguez, V. D.; Méndez-Ramos, J.; Tikhomirov, V. K.; del-Castillo, J.; Yanes, A. C.; Moshchalkov, V. V.; Opt. Mater. 2011, 34, 179.

13. Goodridge, R. D.; Tuck, C. J.; Hague, R. J. M.; Prog. Mater. Sci. 2012, $57,229$.
14. Schmidt, J.; Sachs, M.; Blümel, C.; Winzer, B.; Toni, F.; Wirth, K.-E.; Peukert, Wo.; Procedia Eng. 2015, 102, 550.

15. de Campos, B. M.; Calefi P. S.; Ciuffi, K. J.; de Faria, E. H.; Rocha, L. A.; Nassar, E. J.; Silva, Jorge, V. L.; Oliveira, M. F.; Maia, I. A.; J. Therm. Anal. Calorim. 2014, 115, 1029.

16. Nassar, E. J.; de Sousa, F. J.; de Lima, G. P. A.; Ávila, L. R.; Ciuffi, K. J.; Calefi, P. S.; Mater. Res. 2010, 13, 71.

17. Russell, D. P.; Beaumont. P. W. R.; J. Mater. Sci. 1980, 15, 197.

18. Avlar, S.; Qiao, Y.; Composites, Part A 2005, 36, 624.

19. Liu, X.; Wu, Q.; Eur. Polym. J. 2002, 38, 1383.

20. Zhihui, Y.; Yajie, Z.; Xiaomin, Z.; Jinghua, Y.; Polymer 1998, 39, 547.

21. Xie, S.; Zhang, S.; Liu, H.; Chen, G.; Feng, M.; Qin, H.; Wang, F.; Yang, M.; Polymer 2005, 46, 5417.

22. Illers, K.-H.; Polymer 1977, 18, 551.

23. Cooper, S. J.; Coogan, M.; Everall, N.; Priestnall, I.; Polymer 2001, 42, 10119.

24. Sengupta, R.; Tikku, V. K.; Somani, A. K.; Chaki, T. K.; Bhowmick, A. K.; Radiat. Phys. Chem. 2005, 72, 625.

25. Zydowicz, N.; Chaumont, P.; Soto-Portas, M. L.; J Membrane Sci. 2001, $189,41$.

26. Adame, D.; Beall, G. W.; Appl. Clay Sci. 2009, 42, 545.

27. Liu, H.; Wang, X.; Fang, P.; Wang, S.; Qi, X.; Pan, C.; Xie, G.; Liew, K. M.; Carbon 2010, 48, 721.

28. Pigłowskia, J.; Gancarza, I.; Wlaz’lak, M.; Kammerb, H.-W.; Polymer 2000, 41, 6813 .

29. Adam, F.; Osman, H.; Hello, K. M.; J. Colloid Interface Sci. 2009, 331, 143.

30. Capeletti, L. B.; Baibich, I. M.; Butler, I. S.; Santos, J. H. Z.; Spectrochim. Acta, Part A 2014, 133, 619.

31. Nassar, E. J.; Ávila, L. R.; Pereira, P. F. S.; Nassor, E. C. O.; Ciuffi, K. J.; Calefi, P. S. Quim. Nova 2007, 30, 1567.

32. Nassar, E. J.; Neri, C. R.; Calefi, P S.; Serra, O. A.; J. Non-Cryst. Solids 1999, 247, 124

33. Prado A. G. S.; Faria, E. A.; Quim. Nova 2005, 28, 544.

34. Bandeira, L. C.; Ciuffi, K. J.; Calefi, P. S.; Nassar, E. J.; Silva, J. V. L.; Oliveira, M. F.; Maia, I. A.; Salvado, I. M.; Fernandes M. H. V.; J. Braz. Chem. Soc. 2012, 23, 810.

35. Sharma G.; Narula, A. K.; Sens. Actuators, B 2015, 215, 584.

36. Ryu, S.-J.; Kim, A.; Kim, M. D.; Hong, S. W.; Min, S. S.; Lee, J.-H.; Lee, J.-K.; Jung, H.; Appl. Clay Sci. 2014, 101, 52.

37. Atikins, P.; Shriver, D.; Química inorgânica, 4 ed., Porto Alegre: Bookman, 2008.

38. Bandeira, L. C.; de Campos, B. M.; Calefi, P. S.; Ciuffi, K. J.; Nassar, E. J.; Silva, J. V. L.; Oliveira, M. F.; Maia, I. A.; Journal of Nanostructured Polymers and Nanocomposites 2011, 7/2, 47.

39. Ballistreri, A.; Garozzo, D.; Giuffrida, M.; Impallomeni, G; Montuado, G.; Polym. Degrad. Stabil. 1988, 23, 25.

40. Yu H. H.; Mater. Sci. Eng. 1998, A254, 53.

41. Bezerra, E. B.; Leite, A. M. D; Araújo, E. M.; Mélo T. J. A.; Polímeros 2014, 24, 381.

42. Bansal, B. M.; Damien D. and Koehly, G.; Inorg. Nucl. Chem. Lett. 1969, 5, 509.

43. Sheng, K.; Yan, B.; J. Photochem. Photobiol., A 2009, 206, 140

44. Liu. D.; Shi, Q.; Wang, Z.; Opt. Mater. 2012, 34, 1815.

45. Cunjin, X.; J. Rare Earths 2010, $28,854$.

46. Azevedo, C. B.; Batista, T. M.; de Faria, E. H.; Rocha, L. A.; Ciuffi, K. J.; Nassar, E. J.; J Fluoresc. 2015, 25, 433.

47. Serra, O. A.; Nassar, E. J.; Zapparolli, G.; Rosa, I. L. V.; J. Alloys Compd. 1994, 207-208, 454.

48. de Souza, É. A.; Azevedo, C. B.; Rocha, L. A.; de Faria, E. H.; Calefi, P. S.; Ciuffi, K. J.; Nassar, E. J.; Silva, J. V. L.; Oliveira, M. F.; Maia, I. A.; J. Mater. Res. 2012, 27, 2088.

49. Binnemans, K.; Coord. Chem. Rev. 2015, 295, 1. 
50. Rainho, J. P.; Ananias, D.; Lin, Z.; Ferreira, A.; Carlos, L. D.; Rocha, J.; J. Alloys Compd. 2004, 374, 185

51. Rocha, L. A.; Freiria, J. do C.; Caiut, J. M. A.; Ribeiro, S. J. L.; Messaddeq, Y.; Verelst, M.; Dexpert-Ghys, J.; Nanotechnology 2015, 26, 335604.

52. Dutra, J. D. L.; Bispo, T. D.; Freire, R. O.; J. Comput. Chem. 2014, 35, 772.
53. Reisfeld, R.; Zigansky, E.; Gaft, M.; Mol. Phys. 2004, 102, 1319.

54. Jorgensen, C. K.; Reisfeld, R.; J. Less-Common Met. 1983, 93, 107.

55. Babu, A. B.; Jamalaiah, B. C.; Suhasini, T.; Rao, T. S.; Moorthy, L. R.; Solid State Sci. 2011, 13, 574.

56. Santos, J. G.; Dutra, J. D. L.; Alves, S.; De Sá, G. F.; Da Costa, N. B.; Freire, R. O.; J. Braz. Chem. Soc. 2013, 24, 236.

57. Lima, P. P.; Malta, O. L.; Alves, Jr. S.; Quim. Nova 2005, 28, 805. 